Otterbein University

Digital Commons @ Otterbein

Psychology Faculty Scholarship

Psychology

8-15-2011

\title{
The Relationship between performance in near match-to-sample tasks and fluid intelligence.
}

Meredith C. Frey

Otterbein University, mfrey@otterbein.edu

Follow this and additional works at: https://digitalcommons.otterbein.edu/psych_fac

Part of the Psychology Commons

\section{Repository Citation}

Frey, Meredith C., "The Relationship between performance in near match-to-sample tasks and fluid intelligence." (2011). Psychology Faculty Scholarship. 1.

https://digitalcommons.otterbein.edu/psych_fac/1

This Article is brought to you for free and open access by the Psychology at Digital Commons @ Otterbein. It has been accepted for inclusion in Psychology Faculty Scholarship by an authorized administrator of Digital Commons @ Otterbein. For more information, please contact digitalcommons07@otterbein.edu. 


\title{
The relationship between performance in near match-to-sample tasks and fluid intelligence ${ }^{\text {is }}$
}

\author{
Meredith C. Frey \\ Department of Psychology, Otterbein University, 1 South Grove St., Westerville, $\mathrm{OH} 43081$, United States
}

\section{A R T I C L E I N F O}

\section{Article history:}

Received 3 August 2009

Received in revised form 24 April 2011

Accepted 25 April 2011

Available online 15 August 2011

\section{Keywords:}

Elementary cognitive tasks

Task complexity

Stimulus discrimination

Match to sample

\begin{abstract}
A B S T R A C T
Match-to-sample is a timed task in which a subject is presented with a visual stimulus (the probe) and must select a match to that stimulus (the target) from among an array of distractors. These tasks are frequently employed as tests of basic cognitive abilities and demonstrate consistent correlations with measures of intelligence. In the current study, a match-to-sample task was modified to produce near-match conditions (trials for which no exact match existed). Two factors were manipulated: type of discrepancy between the target and probe (additive or subtractive) and degree of discrepancy between target and probe (1 element or 2 elements). It was hypothesized that introducing near-match conditions would change the processing demands of the task, resulting in increased correlations between decision time and fluid intelligence. Degree and type of discrepancy affected decision times: participants required more time for 2 element discrepancies and additive type discrepancies. Contrary to expectations, increased decision time on a task did not correspond to higher correlations with fluid intelligence. For type, correlations were larger when participants needed to mentally subtract elements from the target in order to make it match the probe (mean $r_{\text {additive }}=.399$; mean $r_{\text {subtractive }}=.451$ ). For degree, correlations were larger for trials where the probe and target differed by one element (mean $r=.470$ ) than for trials where the probe and target differed by two elements (mean $r=.380$ ). These results seem to indicate that the relationship between the complexity of a task and general intelligence is not as straightforward as has been commonly believed.
\end{abstract}

(c) 2011 Elsevier Inc. All rights reserved.
No matter which theory of intelligence one supports, the study of performance on elementary cognitive tasks (ECTs) can inform the understanding of the basic processes involved in more complex cognitive operations. Jensen (1998a) characterized these ECTs as "so simple and so lacking in any specific skill or knowledge content as to greatly limit the possibilities of exactly what the subject must do to perform successfully" (p. 207). They are typically nonverbal, chronometric measures of reaction time, inspection time, working

\footnotetext{
is A portion of this work was supported by Grant No. HD07176 from the National Institute of Child Health and Human Development, Office of Mental Retardation. Thanks to Douglas K. Detterman for his guidance and to Lee Thompson and Heath Demaree for their comments on previous versions of this work.

E-mail address: MFrey@Otterbein.edu.
}

memory capacity, and stimulus discrimination. ECT research has shown moderate correlations between performance on these tasks-defined by speed rather than accuracy-and a general factor (see Jensen, 2006).

These basic cognitive tasks have been combined to predict intelligence, almost as well as traditional intelligence tests predict one another (Detterman, Mayer, Caruso, Legree, Conners \& Taylor, 1992). Additionally, Luo and Petrill (1999) demonstrated that the addition of ECTs to a psychometric battery did not alter the primary criterion validity of intelligence tests- academic achievement- and offered an increased understanding of the underlying mechanisms of intelligence. In fact, Luo, Thompson and Detterman (2006) found moderate correlations (up to $r=.635$ ) between aggregate measures of what they refer to as tasks of basic cognitive processes (TBCPs) and achievement measures (p. 103). 
Given the utility of ECTs in predicting general ability and academic achievement, the primary purpose of this study was to modify an existing ECT to produce a near match-to-sample task. Standard match-to-sample tasks were employed in previous research as a measurement of stimulus discrimination or stimulus coding (Detterman et al., 1992; Luo et al., 2006; Luo \& Petrill, 1999). In Detterman, Luo, and colleagues' administrations, the match-to-sample tasks included the simultaneous presentation of a probe stimulus and an array of six similar stimuli, one of which (the target) matched the probe. Performance on this match-to-sample task (as indicated by mean decision time) correlated strongly $(r=-.70)$ with measures of intelligence in intellectually disabled participants (Detterman et al., 1992, p. 275); additionally, Luo and Petrill (1999, p. 167) found moderate loadings (.42) of this task on a general factor in a sample of elementary school children.

In the previous studies, however, an exact match to the probe stimulus has always been present. It is unknown how participants will respond when no exact match exists. It is possible that processing discrepancy between a probe and target stimulus recruits additional cognitive mechanisms, resulting in increased correlations between performance on the task and general ability. This finding would be consistent with previous research, summarized below, on the relationship between task complexity and general ability.

One of the primary findings in ECT research is that $g$ loadings (or correlations with general ability) increase as a result of higher levels of complexity. However, the notion of complexity is a bit unclear. Jensen (1998b) defined this complexity as "information processing load" (p. 43) or "task difficulty" (p. 44) in his examination of the Hick (1952) paradigm. Hick quantified information processing load as the number of binary decisions (bits) required to complete a task. Consistent with the understanding of complexity as increased information processing, Vernon and Weese (1993) argued more complex tasks place greater demands on the information processing system, resulting in tasks that increasingly approximate complex IQ test items.

However, with the exception of the linear manipulation of complexity (as defined by increasing information load) in the Hick paradigm reaction time tasks, the relationship between task complexity (as defined by task difficulty) and IQ has often been examined across different tasks. For example, choice reaction time, traditionally regarded as a fairly simple cognitive task, tended to correlate roughly -.35 with IQ (see Jensen, 2006 for a thorough account of this research). At the opposite end of the complexity spectrum is working memory, which has correlated up to -.96 with $g$ (Colom, Rebollo, Palacios, Juan-Espinosa \& Kyllonen, 2004; Kyllonen \& Christal, 1990). Vernon and Jensen (1984) examined a variety of ECTs and found that as tasks increased in complexity-in this case increased complexity was operationally defined as increased mean response latency-the correlations between these tasks and a general factor extracted from a battery of aptitude tests increased accordingly. A potential criticism of this understanding of complexity has emerged in the striking differences between the tasks themselves: Vernon and Jensen employed a variety of reaction time and working memory tasks, which differed in many ways. For example, while reaction time tasks can be accomplished with minimal reliance on verbal or numeric processing, working memory tasks often use letters or numbers as stimuli. Furthermore, traditional working memory tasks have a greater range of item difficulty than is typically found in reaction time tasks, which could also contribute to higher correlations. Simply stated, researchers have no way of knowing if changes in correlations with general ability were due solely to complexity or to other differences between the tasks. If task complexity determined these correlations, then systematically altering a single task should produce changes in the correlations with IQ, and these changes should be predictable (e.g., correlations between decision time and intelligence test scores should increase as the complexity of the task increases).

Unfortunately, previous attempts to linearly manipulate complexity within traditionally employed ECTs, match-tosample and choice reaction time, have met with little success. A match-to-sample task modified to include increasing numbers of alternatives, similar to a Hick paradigm, yielded expected increases in decision time for participants, but not significant changes in the task-IQ correlations (Frey \& Detterman, 2002). Similarly, a choice reaction time task modified to include some additional numeric judgment-odd/ even or low/high-yielded increases in decision times, but significant changes in the task-IQ correlations were absent (Frey \& Detterman, 2004).

It was possible that subject selection procedures-all participants were students at a selective university-and subsequent restriction of range of IQ in the Frey and Detterman work served to disguise task-IQ correlations. Certainly, an increased range of ability is warranted in the current study. However, theirs was not the only research on the relationship between task complexity and intelligence. Indeed, even the expected changes in correlations between reaction time (RT) and general ability across varying degrees of information load have been elusive. Sheppard (2008) examined 50 years of published results in the domain of intelligence and speed of information processing, and, while she reported a trend for $\mathrm{RT} \times$ intelligence correlations to be higher with increased bits of information processing, the mean correlations reported were less clear. For example, the mean correlations across studies between RT and intelligence at 2, 3, and 5 bits were identical ( $r=.28$; Sheppard, 2008, p. 536). Indeed, when discussing the change in RT-IQ correlation with increasing numbers of bits, Jensen (1987, p. 165) called the finding "a rather weak and erratic phenomenon".

For the current study, then, a different type of complexity manipulation was examined within a match-to-sample task. Previous researchers have made assertions about complexity across different types of tasks, or by increasing the number of alternatives within a single task. The former understanding of complexity is problematic, as it requires generalizations across fundamentally different tasks. Support for the latter understanding of complexity has been inconsistent; generally the expected increases in decision time are present, but the expected changes in correlations with intelligence are absent. Therefore, it was hypothesized that changing a task from a simple match-to-sample task to one where no exact match exists would result in the necessary additional processing to increase both decision times and task-IQ correlations. Additionally, if processing discrepancy between a probe and 
target stimulus required additional cognitive resources, it was expected that the degree of discrepancy between a probe and target stimulus would affect decision times and task-IQ correlations.

\section{Method}

\subsection{Participants}

\subsubsection{University sample}

Fifty-two undergraduates from a selective, private research university were recruited as subjects. One participant did not complete one of the tasks (Minus 2) but the data for the remaining tasks were retained. Participants were 28 males, 23 females, and 1 who did not identify sex; they ranged in age from 18 to 23 years $(M=19.18, S D=1.07)$. The students were members of a general psychology subject pool and received course credit in exchange for their participation.

\subsubsection{Community sample}

In order to increase the range of IQ scores in the sample, 36 normal adult volunteers from communities in Northeast Ohio were also recruited to serve as subjects. Participants in this sample were 18 males, 17 females, and 1 who did not identify sex; they ranged in age from 18 to 60 years $(M=35.83$, $S D=12.16)$. They received a $\$ 10$ gift certificate to a local entertainment venue or retail establishment in exchange for their participation.

\subsection{Materials}

\subsubsection{The CAT}

The Cognitive Abilities Test (Detterman, 1988) was used to test subjects' performance on a battery of computer-based match-to-sample tasks (MTS; referred to as "stimulus discrimination" or $S D$ in the test manual). Subjects received the standard MTS task (MTS-S), a Plus 1 task (MTS-P1), a Minus 1 task (MTS-M1), a Plus 2 task (MTS-P2), and a Minus 2 task (MTS-M2). The differences between these tasks have been detailed below; more generally, to produce the nearmatch trials, illuminated square(s) were either added to or subtracted from the probe stimulus, a $4 \times 4$ partially filled matrix, resulting in an imperfect match to the target stimulus. For all of these tasks, in order to start each trial, the subject was required to press and hold down the spacebar (home key). Once the home key was depressed, the patterns in the probe, target, and remaining alternatives appeared. Subjects were instructed to find the target, release the home key, and indicate the position of the target by pressing a number (1-6) on a standard keyboard. When the participant released the home key, the screen cleared; this ensured participants had actually found a match before releasing the home key. Decision time was recorded as the time participants depressed the home key. If participants released the home key in error, they were instructed to press the spacebar again and the trial stimuli reappeared, resulting in multiple decision times for a single trial. In these circumstances, the multiple decision times were summed.

Each task contained 8 practice and 72 actual trials. Participants received auditory feedback on each trial; correct trials were followed by a high-pitched beep and incorrect trials were followed by a low-pitched buzz. Error trials were re-administered for each task.

\subsubsection{Match-to-sample standard (MTS-S)}

The standard match-to-sample task window (see Fig. 1) consisted of simultaneous presentation of a probe stimulus (the pattern at the top center of the screen) and an array of six alternatives below the probe. The alternatives were numbered (from left to right) 1 to 6 . One of the six choices (the target) was an exact match to the probe.

\subsubsection{Match-to-sample plus 1 (MTS-P1)}

This task was designed to be as similar as possible to the standard match-to-sample condition, however, in MTS-P1 trials, no exact match to the probe stimulus existed in the numbered array below it. One pattern in the numbered array closely resembled the probe stimulus. Participants had to select the pattern that most closely resembled the probe. The probe and target differed by exactly one illuminated square (participants mentally added one illuminated square to the target to make the pattern exactly match the probe; see Fig. 2).

\subsubsection{Match-to-sample minus 1 (MTS-M1)}

The target in MTS-M1 tasks was also one square different than the probe. However, in this task, participants were required to mentally subtract one illuminated square from the target to obtain an exact match to the probe (see Fig. 3).

\subsubsection{Match-to-sample plus 2 (MTS-P2)}

This task was a near-match condition ( similar to MTS-P1). However, in this case participants mentally added two squares to the target to make it exactly match the probe. MTS-P2 was added to the task battery to determine if degree of discrepancy (one square vs. two squares) affected decision times (or correlations with general ability) of the Plus task type (see Fig. 4).

\subsubsection{Match-to-sample minus 2 (MTS-M2)}

A fourth task near-match (MTS-M2) was included to assess the effects of degree of discrepancy on key variables in

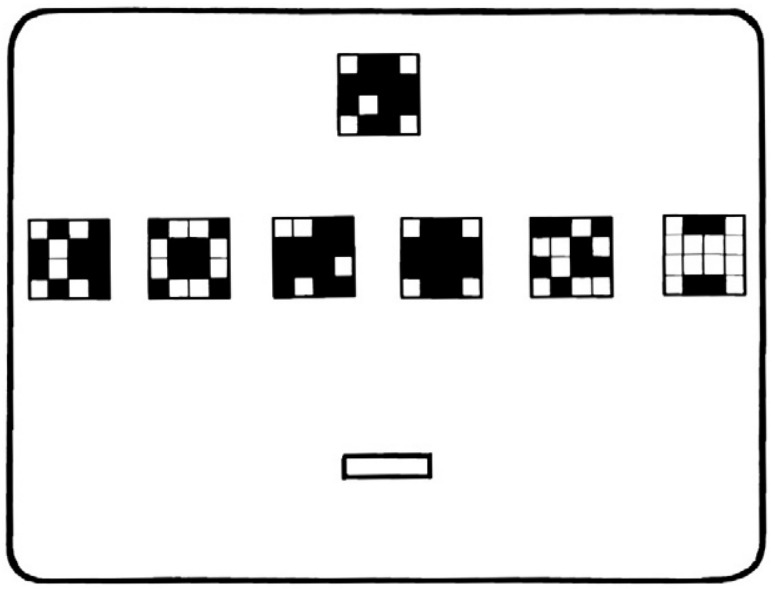

Fig. 1. A typical MTS task in the CAT battery. The target is in the \#4 position. 


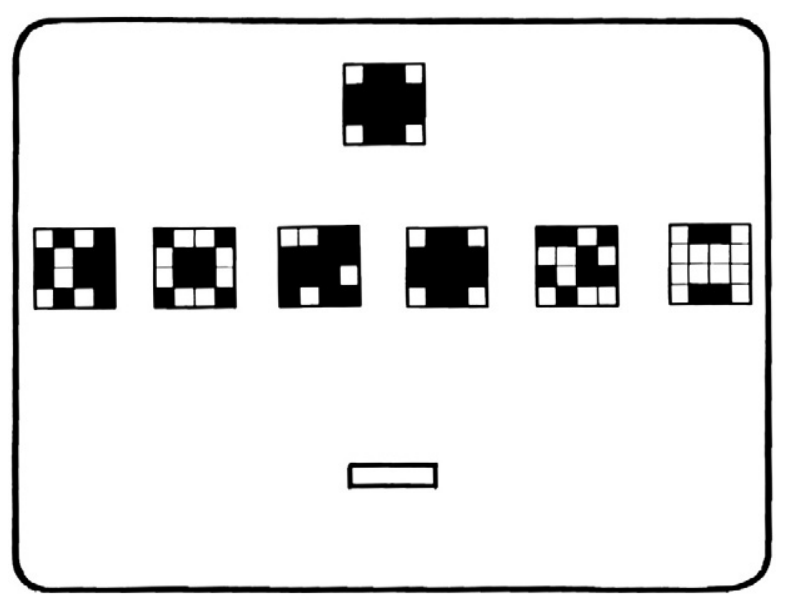

Fig. 2. MTS Plus 1 (MTS-P1). The target is in the \#4 position.

the Minus task type. MTS-M2 was also a near-match condition (similar to MTS-M1) where two squares were mentally subtracted from the target to obtain an exact match to the probe (see Fig. 5).

\subsubsection{Raven's APM}

The Advanced Progressive Matrices (APM; Raven, 1962) was the primary means of measuring intelligence in subjects from both the community and university samples. The APM, a 36item nonverbal test of fluid reasoning ability, was selected for three reasons: 1) it was available for subjects outside the university subject pool, 2) it has been considered the purest measure of fluid ability (Marshalek, Lohman \& Snow, 1983), and 3 ) it has been widely used as an estimate of general intelligence.

Each problem in the APM contained an incomplete $3 \times 3$ matrix (i.e. 3 rows, 3 columns). The individual elements of the matrix formed a specific pattern, with the bottom right element missing. Subjects were presented with eight alternatives and asked to select the element that best completed the pattern.

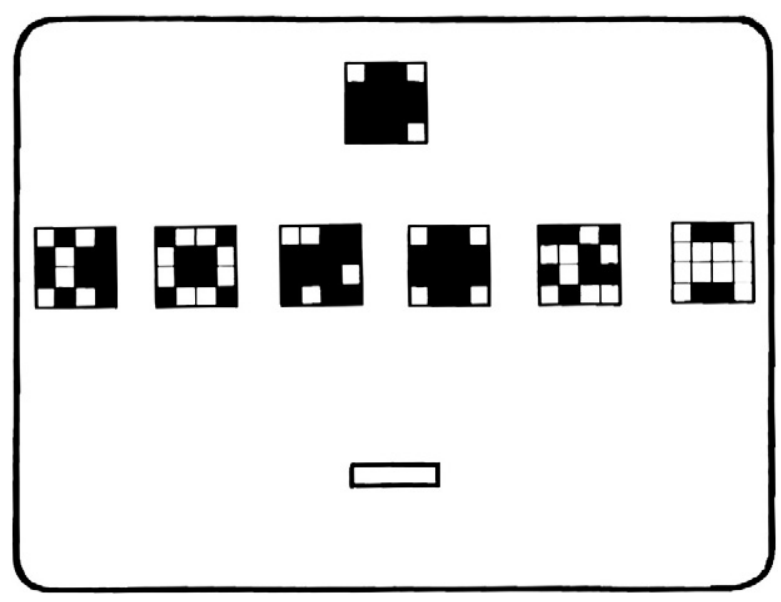

Fig. 3. MTS Minus 1 (MTS-M1). The target is in the \#4 position.

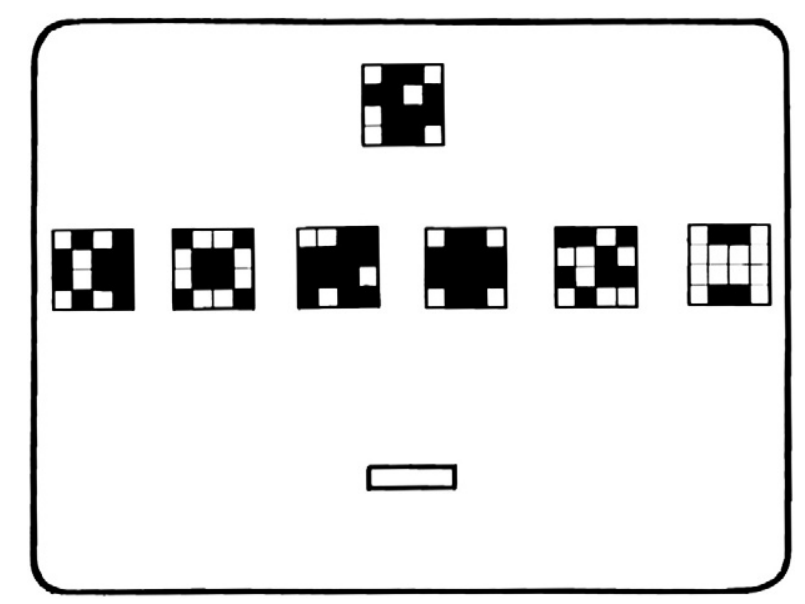

Fig. 4. MTS Plus 2 (MTS-P2). The target is in the \#4 position.

\subsection{Procedure}

All subjects completed an untimed administration of the APM before beginning the CAT tasks, most immediately before the CAT. In some cases however, there was as much as a week or more between administration of the Raven's and the CAT battery. These individuals experienced this delay for one of two reasons: 1 ) subjects had taken the APM as part of another study and data were obtained directly from that experimenter to avoid test-retest contamination, or 2) subjects took an extended amount of time completing the APM and needed to complete the CAT battery at a later date due to scheduling conflict. The experimenter gave oral instructions on the APM, and completed the first item with the subjects to demonstrate how to solve the problems. All subjects completed the CAT with standard keyboard administration. Participants were seated approximately 40 inches from the monitor. All university students were administered the test on a standard desktop computer, while most (27 of 36) community volunteers completed the CAT on a laptop.

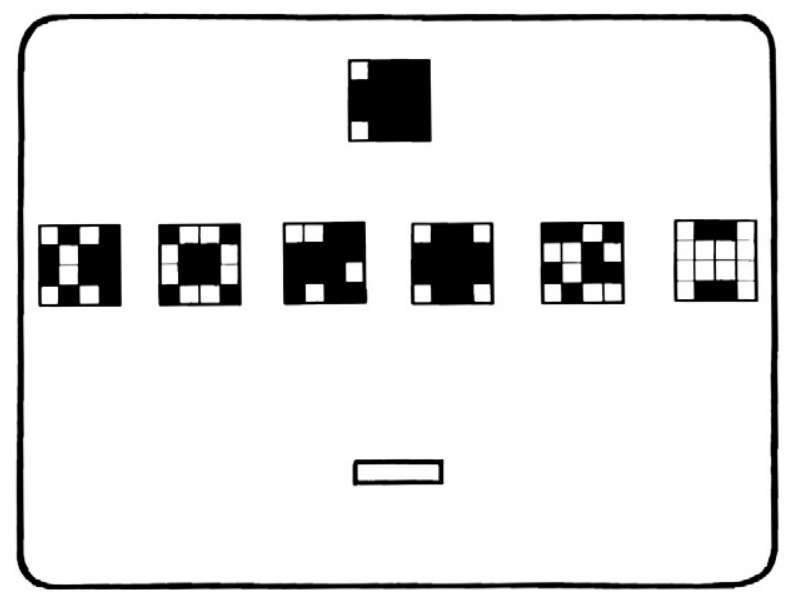

Fig. 5. MTS Minus 2 (MTS-M2). The target is in the \#4 position. 
However, the monitor size was the same across apparatuses (15 in.), to ensure task equality.

Subjects were administered the CAT tasks in one of two orders. In both orders, MTS-S was administered first to allow subjects to become acclimated to the procedure of the matchto-sample tasks before the near-match conditions. Both orders also placed the more discrepant (Plus 2 and Minus 2 ) tasks before the less discrepant. This was done to ensure that differences in decision times were not the result of fatigue effects. Since all subjects received the MTS-S condition first, they were considered sufficiently familiar with the procedures to avoid practice effects influencing the later tasks. Roughly half of the participants received the order MTS-S, MTS-P2, MTS-M2, MTS-P1, and MTS-M1. The other half received the order MTS-S, MTS-M2, MTS-P2, MTS-M1, and MTS-P1.

\section{Results}

\subsection{Preliminary analyses}

All APM scores were converted to an IQ scale using transformation data from the test manual (Raven, Raven \& Court, 1998, p. APM 102). The data were examined for influential cases. A conservative multiple regression approach to identifying influential cases was selected. IQ was regressed on mean decision time (DT) for each task. A case was considered unduly influential if its removal changed the unstandardized b-coefficient of this regression by greater than one standard error. No cases met this criterion, so all data were retained for further analysis.

\subsection{Order effects}

Independent samples $t$-tests were conducted on the mean decision times and DT $\times$ IQ correlations for each of the five tasks to determine if there were any effects of the order of task presentation on outcomes. All $t$-tests were non-significant (all $p>.100$ ), indicating that it was appropriate to collapse the data across order for the remaining analyses.

\subsection{IQ differences}

The overall mean IQ, as measured by the APM, was 109.9 $(S D=13.6)$. The mean IQ for the university sample was 112.2 $(S D=13.1)$, while the mean IQ for the community sample was $106.5(S D=13.8)$. Although both samples were above the normative mean of IQ this difference between groups was statistically significant $(p=.05)$. Furthermore, the age difference between the two groups was substantial. Therefore, sample differences on mean DTs, standard deviations of DT, and correlations with IQ were examined.

\subsection{Mean decision times (mean DTs)}

Mean decision times for all tasks, corrected for outliers, were computed for both the university and community samples, as well as for the groups combined (see Table 1). In order to correct for outliers within participants, any trial decision time that was greater than three standard deviations from the original mean was reset to three standard de-
Table 1

Mean decision times (DTs; in seconds) and standard deviations (in parentheses) corrected for outliers.

\begin{tabular}{llll}
\hline Task & University sample & Community sample $^{\mathrm{b}}$ & Overall $^{\mathrm{c}}$ \\
\hline MTS-S & $1.817(0.373)$ & $2.348(0.721)$ & $2.034(0.599)$ \\
MTS-M1 & $2.200(0.539)$ & $2.957(1.066)$ & $2.510(0.876)$ \\
MTS-M2 & $3.472(1.031)$ & $4.133(1.724)$ & $3.746(1.391)$ \\
MTS-P1 & $2.513(0.687)$ & $3.348(1.374)$ & $2.854(1.099)$ \\
MTS-P2 & $4.364(1.249)$ & $5.081(2.304)$ & $4.657(1.782)$ \\
\hline
\end{tabular}

${ }^{\mathrm{a}} \mathrm{N}=51 ;{ }^{\mathrm{b}} \mathrm{N}=36 ;{ }^{\mathrm{c}} \mathrm{N}=87$.

viations. Following this adjustment, a mean and standard deviation were recalculated. For all groups, the mean DTs followed the same pattern (from shortest DT to longest): MTS Standard (MTS-S), MTS Minus 1 (MTS-M1), MTS Plus 1 (MTSP1), MTS Minus 2 (MTS-M2), MTS Plus 2 (MTS-P2). The pattern of these decision times provided evidence in support of the stated hypothesis: the tasks where no exact match was present yielded longer decision times, and the degree of disparity between the probe and the target systematically influenced DTs. The community sample had significantly longer DTs for all tasks except for MTS-P2 $(p=.06)$, which was to be expected according to the negative correlation between IQ and DT. Because the groups differed significantly on key variables, notably age and IQ, the DT analyses were conducted first on the combined groups, then on each of the individual samples.

\subsubsection{Combined sample}

A repeated measures ANOVA demonstrated a significant effect of task type on mean DT $(F(4,344)=238.871 ; p<.001)$. Subsequent pairwise comparisons, adjusted for multiple contrasts using a Bonferroni correction, revealed that each task's mean DT was significantly different from all others (all $p<.001$ ). Repeating the analysis on each subsample produced the same results.

\subsubsection{Effect size}

The effect sizes for all analyses above were large, ranging from partial $\eta^{2}$ of .657 to .812 . This result indicated that the majority of the variance in mean decision times was accounted for by task type.

\subsection{Mean decision time $\times I Q$ correlations}

The mean DT $\times$ IQ correlations for all tasks are presented in Table 2.

Table 2

Mean DT $\times$ IQ correlations.

\begin{tabular}{llll}
\hline Task & University sample $^{\mathrm{a}}$ & Community sample $^{\mathrm{b}}$ & Overall $^{\mathrm{c}}$ \\
\hline MTS-S & -.204 & $-.398^{*}$ & $-.358^{* *}$ \\
MTS-M1 & $-.424^{* *}$ & $-.496^{* *}$ & $-.482^{* *}$ \\
MTS-M2 & $-.342^{* *}$ & $-.509^{* *}$ & $-.421^{* *}$ \\
MTS-P1 & $-.392^{* *}$ & $-.410^{*}$ & $-.459^{* *}$ \\
MTS-P2 & $-.377^{* *}$ & -.284 & $-.340^{* *}$ \\
\hline
\end{tabular}

${ }^{\mathrm{a}} \mathrm{N}=51-52 ;{ }^{\mathrm{b}}=36 ;{ }^{\mathrm{c}} \mathrm{N}=87-88$.

* Correlation is significant at .05 level (2-tailed).

** Correlation is significant at .01 level (2-tailed). 


\subsubsection{Sample differences}

The individual task correlations with IQ in the university sample were compared to the corresponding tasks in the community sample to determine which tasks, if any, yielded different correlations across groups. A Fisher's z-test for independent correlations was performed on each of the task pairings. No task was found to have significantly different correlations with IQ across groups. Because no betweengroup differences were found, the remaining correlation analyses were performed only on the combined groups to maximize statistical power.

\subsubsection{Task differences}

MTS-P2 had the lowest overall correlation with IQ, followed by MTS-S, MTS-M2, MTS-P1, and MTS-M1. The pattern of these correlations, presented in Column 3 of Table 2, was examined to determine whether or not the tasks varied significantly in terms of their respective correlations. Several contrasts were found to be statistically significant (see Table 3). Notably, the DTs in the minus type tasks yielded significantly higher correlations with IQ than the plus type tasks. Furthermore, there was a trend for the less discrepant (MTS-M1 and MTS-P1) tasks to correlate more highly with IQ than the more discrepant (MTS-M2 and MTS-P2) tasks.

\subsection{Standard deviations of decision times (SDDT)}

Jensen (1998a) has suggested intraindividual variability in decision times as a good predictor of general intelligence, often higher than the decision times themselves (p. 226). That is, participants with higher levels of intelligence would be expected to have lower variability in decision times within a task. In order to test this, participants' standard deviations of decision times (once DTs were corrected for outliers as described above), were computed and analyzed for mean differences and for correlations with fluid intelligence.

\subsubsection{Task differences in SDDT}

Standard deviations for each task (overall and separated by subgroup) are presented in Table 4. A split plot ANOVA yielded a significant effect of task type on intraindividual variability (Wilks' Lambda $=.257, F(4,82)=59.140, p<.001$ ). This effect was quite large $\left(\eta_{\mathrm{p}}^{2}=.743\right)$. There was a significant but small effect of group $(F(1,85)=3.957, p=.05$, $\left.\eta_{\mathrm{p}}^{2}=.084\right)$, but no significant group $\times$ task interaction $(p=.123)$.

Table 3

Significant differences in decision time $\times$ IQ correlations across tasks.

\begin{tabular}{lll}
\hline Contrast & $t$ & $p$ \\
\hline MTS-M1 $>$ MTS-S & 2.67 & $<.01$ \\
MTS-P1 $>$ MTS-S & 1.78 & $<.05$ \\
MTS-M1 $>$ MTS-P2 & 2.65 & $<.01$ \\
MTS-M2 > MTS-P2 & 1.78 & $<.05$ \\
MTS-P1 $>$ MTS-P2 & 2.06 & $<.05$ \\
\hline
\end{tabular}

Note: All contrasts computed using $t$-tests for differences between related correlations (see Cohen \& Cohen, 1983, p. 57).
Table 4

Standard deviation of decision times corrected for outliers.

\begin{tabular}{llll}
\hline Task & University sample $^{\mathrm{a}}$ & Community sample $^{\mathrm{b}}$ & Overall $^{\mathrm{c}}$ \\
\hline MTS-S & 0.65 & 0.86 & 0.74 \\
MTS-M1 & 1.03 & 1.41 & 1.19 \\
MTS-M2 & 1.86 & 2.03 & 1.93 \\
MTS-P1 & 1.27 & 1.70 & 1.45 \\
MTS-P2 & 2.49 & 2.85 & 2.64 \\
\hline
\end{tabular}

${ }^{\mathrm{a}} \mathrm{N}=51 ;{ }^{\mathrm{b}} \mathrm{N}=36 ;{ }^{\mathrm{c}} \mathrm{N}=87$.

\subsubsection{SDDT $\times I Q$ correlations}

Correlations between standard deviations of decision times and IQ are presented in Table 5. Once again, a Fisher's z-test for differences between independent correlations indicated no significant differences across groups (all $p>.15$ ), indicating it was acceptable to collapse across groups to maximize statistical power. Of the ten possible contrasts, only one yielded a significant difference between SDDT $\times$ IQ correlations across tasks. The SDDT of participants in the Plus 1 condition was significantly more correlated with IQ than the SDDT of participants in the Plus 2 condition $(t=-2.13$, $p<.05$ ).

\subsection{Statistical power}

The exploratory nature of this study made a priori power analysis nearly impossible, as the magnitudes of the correlations with IQ and size of differences between correlations were unknown in advance. Observed power was acceptable for analyses of mean differences (all >.90). When comparing correlations with IQ across tasks, in order to obtain an acceptable $(>.80)$ level of power, over 1500 subjects would have been required for some of the comparisons (i.e. MTS-S/ MTS-P2; MTS-M1/MTS-P1). Additionally, when looking at the size of the differences ( $1 \%$ and $2 \%$ increment in explained variance, respectively), it was unlikely that the results would have been theoretically meaningful.

\section{Discussion}

Detterman (1987a) advocated for the creation of a systematic program of research to investigate a number of ECTs in hopes of furthering the understanding of the basic mechanisms of intelligence. Luo and Petrill (1999) also promoted the inclusion of ECT research in our understanding of intelligence, precisely because these tasks tend to be

Table 5

Standard deviation of DT $\times$ APM Correlations.

\begin{tabular}{llll}
\hline Task & University sample $^{\mathrm{a}}$ & Community sample $^{\mathrm{b}}$ & Overall $^{\mathrm{c}}$ \\
\hline MTS-S & -.183 & -.293 & $-.284^{* *}$ \\
MTS-M1 & -.272 & $-.443^{* *}$ & $-.395^{* *}$ \\
MTS-M2 & $-.345^{*}$ & -.204 & $-.282^{* *}$ \\
MTS-P1 & $-.291^{*}$ & $-.446^{* *}$ & $-.400^{* *}$ \\
MTS-P2 & $-.421^{* *}$ & $-.122^{*}$ & $-.252^{*}$ \\
\hline
\end{tabular}

${ }^{\mathrm{a}} \mathrm{N}=51-52 ;{ }^{\mathrm{b}} \mathrm{N}=36 ;{ }^{\mathrm{c}} \mathrm{N}=87-88$.

* Correlation is significant at .05 level (2-tailed).

** Correlation is significant at .01 level (2-tailed). 
subject to increased experimental control as compared to traditional measures and may "resolve to a certain extent the problem of a circular definition of intelligence" (p. 157). However, others, notably Ceci (1992), asserted that even elementary tasks are not necessarily simple, and the underlying processes of these tasks may not be well understood. Indeed, one of the common assertions about ECTs, that increases in a task's complexity result in increased task-IQ correlations, appears to have remarkably thin support in the literature. It is with the goal of increasing our understanding of ECTs and their relationship to intelligence in mind that the present study, containing a systematic manipulation of the complexity of a match-to-sample task, was conducted.

\subsection{Effects of type of discrepancy and degree of discrepancy}

Partially consistent with the stated hypothesis, introducing a near-match condition sometimes resulted in increased mean DT and DT $\times$ IQ correlations for a match-to-sample task (see MTS-M1 vs. MTS-S and MTS-P1 vs. MTS-S). Interestingly, correlations between DT and IQ for Minus tasks (MTS-M1 and MTS-M2) tended to be larger than these same correlations for the Plus tasks (MTS-P1 and MTS-P2). It is important to note that these tasks were identical in every way except for the mental operation subjects performed in order to resolve the discrepancy between a probe and target stimulus. The Minus tasks required that participants mentally subtract element(s) from the target to make it exactly match the probe, while the Plus tasks required that participants mentally add element(s) to the target to make it exactly match the probe.

According to a system theory of intelligence (see Detterman, 1987b), tasks which are designed to have identical input and output processes but demonstrate predictable differences in task-IQ correlations would likely be accessing different basic cognitive mechanisms. Simply stated, a task that looks a lot like another but yields a higher correlation between performance and a more general IQ measure is likely measuring an additional cognitive component of intelligence. The results presented here indicate that the Minus tasks may be accessing additional cognitive processes than what are necessary to successfully complete Plus tasks. In order to be more specific about the processes involved, future research should examine this type of manipulation in other tasks.

Correlations between DT and IQ for tasks where the probe and target differed by only one element (MTS-M1 and MTSP1) tended to be larger than these same correlations for tasks where the probe and target differed by two elements (MTSM2 and MTS-P2). Although the exact nature of the specific processes in intelligence remains unknown, it is possible that some aspect of performance on the ECTs studied here involves learning the rules of the tasks themselves. This explanation is particularly likely when one takes into account the presentation of tasks in the present study. Although all participants received a standard match-to-sample task first and were therefore considered sufficiently practiced in the task, more discrepant trials occurred before less discrepant trials-that is Plus 2 and Minus 2 tasks always preceded Plus 1 and Minus 1. Possibly, the more discrepant tasks provided an opportunity to learn the rules that governed responses on the less discrepant tasks, resulting then in longer decision times for the more discrepant trials but not in higher task-IQ correlations. This was also supported by standard deviations of decision times (SDDT) across tasks; participants were more variable in the Plus 2 and Minus 2 tasks, but their SDDT-IQ correlations tended to be higher for the Plus 1 and Minus 1 tasks. If rule acquisition was really at work here, it is important to note that the experience-dependent context operates somewhat differently than verbal instruction. Pilot testing for the near-match tasks indicated that precise verbal instructions on the task rules in near match-conditions (i.e., "For the following tasks, you must add one square to one of the six patterns at the bottom of the screen in order to make it exactly match the pattern at the top of the screen.") did not affect performance.

\subsection{Relationship between decision time and task-APM correlations}

The most interesting finding in the present study was the difference between difficulty as measured by decision time and difficulty as measured by task-IQ correlations. Previous research indicated that, as task decision times increased, taskIQ correlations increased as well. The current study found the opposite. More discrepant tasks (i.e. MTS-P2 and MTS-M2) had longer decision times than their less discrepant counterparts (MTS-P1 and MTS-M1) but lower correlations with fluid intelligence. Furthermore, plus type discrepancies (MTS-P1 and MTS-P2) had longer decision times and lower correlations with IQ than their minus type counterparts (MTS-M1 and MTS-M2). The reason for this finding is unknown and replication is warranted. Certainly, one may argue that, whatever task complexity is, it has not been captured by the manipulation of discrepancy between a probe and target stimulus in a match-to-sample task. However, as complexity is sometimes operationally defined as an increase in response latency (Vernon \& Jensen, 1984), the results presented here at the very least indicate that caution should be employed when making the assertion that increased complexity results in increased correlations between a task and IQ.

\subsection{Future directions}

The presence of some differences in task-IQ correlations in these near-match tasks is exciting and may be a preliminary step in uncovering a basic component of intelligence, and it seems this study raises some interesting questions about previously understood conceptions of task complexity. However, future research requires a fully randomized task presentation to adequately address the effect of degree of discrepancy on task-IQ correlations. Indeed it would be best to present trials representative of all five task types randomly within one task. This method would allow for a more precise examination of the hypotheses in that subjects would not be able to acquire the rules in the first few trials and apply them to the rest of the task, confident that those rules do not change. Additionally, more tasks for which there can be a systematic manipulation of the task's complexity need to be uncovered. 


\section{References}

Ceci, S. J. (1992). Is less really more? Using microlevel sensory and cognitive measures to predict macrolevel cognitive outcomes. American Journal on Mental Retardation, 97, 287-290.

Cohen, J., \& Cohen, P. (1983). Applied multiple regression/correlation analysis for the behavioral sciences (Second edition). Hillsdale, NJ: Lawrence Erlbaum Associates.

Colom, R., Rebollo, I., Palacios, A., Juan-Espinosa, M., \& Kyllonen, P. C. (2004). Working memory is (almost) perfectly predicted by $g$. Intelligence, 32, 277-296.

Detterman, D. K. (1987a). Intelligence and reaction time. In P. A. Vernon (Ed.), Speed of information processing and intelligence (pp. 177-200). Norwood, NJ: Ablex.

Detterman, D. K. (1987b). Theoretical notions of intelligence and mental retardation. American Journal of Mental Deficiency, 92, 2-11.

Detterman, D. K. (1988). Cognitive Abilities Test.

Detterman, D. K., Mayer, J. D., Caruso, D. R., Legree, P. J., Conners, F. A., \& Taylor, R. (1992). Assessment of basic cognitive abilities in relation to cognitive deficits. American Journal on Mental Retardation, 97. $251-286$

Frey, M. C., \& Detterman, D. K. (2002, December). Task complexity and stimulus discrimination. Paper presented at the meeting of the International Society for Intelligence Research. Nashville, TN: Vanderbilt University.

Frey, M. C., \& Detterman, D. K. (2004, December). Response complexity and IQ. Paper presented at the meeting of the International Society for Intelligence Research New Orleans, LA.

Hick, W. E. (1952). On the rate of gain of information. The Quarterly Journal of Experimental Psychology, 4, 11-26.
Jensen, A. R. (1987). Individual differences in the Hick paradigm. In P. A. Vernon (Ed.), Speed of information processing and intelligence (pp. 101-175). Norwood, NJ: Ablex.

Jensen, A. R. (1998a). The g factor: The science of mental ability. Westport, Connecticut: Praeger.

Jensen, A. R. (1998b). The suppressed relationship between IQ and the reaction time slope parameter of the Hick function. Intelligence, 26, 43-52.

Jensen, A. R. (2006). Clocking the mind: Mental chronometry and individual differences. Amsterdam: Elsevier.

Kyllonen, P. C., \& Christal, R. E. (1990). Reasoning ability is (little more than) working-memory capacity?! Intelligence, 14, 389-433.

Luo, D., \& Petrill, S. A. (1999). Elementary cognitive tasks and their roles in $g$ estimates. Intelligence, 27, 157-174.

Luo, D., Thompson, L. A., \& Detterman, D. K. (2006). The criterion validity of tasks of basic cognitive processes. Intelligence, 34, 79-120.

Marshalek, B., Lohman, D. F., \& Snow, R. E. (1983). The complexity continuum in the radex and hierarchical models of intelligence. Intelligence, 7(2), 107-127.

Raven, J. C. (1962). Advanced Progressive Matrices Set II. London: H.K. Lewis \& Co..

Raven, J., Raven, J. C., \& Court, J. H. (1998). Manual for Raven's Progressive Matrices and Vocabulary Scales. Section 4: The Advanced Progressive Matrices. San Antonio, TX: Harcourt Assessment.

Sheppard, L. D. (2008). Intelligence and speed of information-processing: A review of 50 years of research. Personality and Individual Differences, 44 , 533-549.

Vernon, P. A., \& Jensen, A. R. (1984). Individual and group differences in intelligence and speed of processing. Personality and Individual Differences, 5, 411-423.

Vernon, P. A., \& Weese, S. E. (1993). Predicting intelligence with multiple speed of information-processing tests. Personality and Individual Differences, 14, 413-419. 